\title{
Correlation between Recrystallization Texture and Heterogeneities in Deformed Structure of an Electrical Steel by Electron Back-scatter Diffraction
}

\author{
Wei-Chih HSU, ${ }^{1)}$ Liuwen CHANG, ${ }^{1 *}$ Pei-Ling SUN, ${ }^{1)}$ Newjin $\mathrm{HO}^{1)}$ Po-We KAO ${ }^{1)}$ and I-Ching HSIAO ${ }^{2)}$ \\ 1) Department of Materials and Optoelectronic Science, National Sun Yat-sen University, Kaohsiung, 80424 Taiwan, R.O.C. \\ 2) Department of Steel Research and Development, China Steel Corporation, Kaohsiung, 81233 Taiwan, R.O.C.
}

(Received on March 23, 2015; accepted on June 11, 2015)

\begin{abstract}
The correlation of recrystallization nucleation and the deformation microstructure in an electrical steel was studied by examining micro-indenter marked specimens with electron back-scatter diffraction (EBSD). The deformed grains were classified according to the density of high-angle boundaries in the grain interiors, and the local misorientation of substructure was characterized by using both the kernel average misorientation (KAM) and the high misorientation region (HMR) analyses. The EBSD observation confirmed that recrystallization was preferentially evolved from areas having high local misorientation revealed by HMRs. The recrystallization nuclei in partially recrystallized specimen were observed to be directly related to the high-KAM areas in the deformed grains. The orientation distribution of the areas having high local misorientation in the cold-rolled structure, which fulfill both criteria of high-KAM and HMR, was found to be associated with the texture of recrystallized grains.
\end{abstract}

KEY WORDS: electrical steel; cold rolled; recrystallization; electron back-scatter diffraction; microtexture.

\section{Introduction}

The evolution of the crystallographic texture in lowcarbon steels from a deformed state to a full recrystallization (RX) state upon annealing is an issue with both academic and practical importance. ${ }^{1-4)}$ Normally, the dominant components in the cold-rolled (CR) texture, $\{\mathrm{hkl}\}<110>$, vanish after RX. Conversely, the RX texture, consisting of varying intensities of $\{001\}<\mathrm{uvw}>,\{111\}<\mathrm{uvw}>$ and $\{011\}<100>$ components, develops during annealing. The recrystallized grains are evolved from the CR structure. Though, experimental results indicated that the dominant components in the RX texture were present in rather low populations in the CR structure. ${ }^{3,4)}$ Considering the fact that profoundly differences of the RX textures were observed for many steels regardless their CR textures were rather similar, the success of a subgrain to develop into an RX grain must depend on the heterogeneities in the deformation microstructure. It is therefore valuable to characterize the structural heterogeneities and identify the potential subgrains, which can develop into RX nuclei, in the CR structure.

The recrystallized nuclei were found to evolve from fast growing subgrains surrounded by high angle boundaries (HABs). ${ }^{5,6)}$ It is believed that a successful nucleation event requires the formation of mobile HABs that can sweep the surrounding deformed matrix. However, for a subgrain to become a growing new grain, possession of HABs is a nec-

\footnotetext{
* Corresponding author: E-mail: 1wchang@mail.nsysu.edu.tw DOI: http://dx.doi.org/10.2355/isijinternational.ISIJINT-2015-159
}

essary but not sufficient criterion. Moreover, the subgrain must have sufficient energy advantage, the difference in stored energy across the boundary, to drive the boundary into the deformed matrix. Based on the aforementioned criteria for RX nucleation, an identification of the subgrains enclosing by HABs and located in the high stored energy region should provide useful clues for the prediction of RX textures.

Heterogeneities are a general characteristic of deformed microstructures, which exist in many forms and over many length scales. Orientation image mapping (OIM) based on electron back-scatter diffraction (EBSD) can provide spatial distribution of crystal orientations over a large area. Therefore, EBSD investigations are well suited for characterizing the heterogeneities in deformed structures. Both HABs and difference in stored energy across the boundary are related to local misorientation in the deformed structure, which could be analyzed by using EBSD with proper step size. It has been shown that the local misorientation distribution in CR structures could be characterized using kernel average misorientation (KAM) method. ${ }^{7)}$ This study has thus attempted to screen the substructures partly or entirely bounded by HABs according to their KAM values. The pixels with high-KAM values may be related to the subgrains enclosing by HABs in the deformed structure.

The distribution of stored energy in a deformed matrix is related to regions with different populations of low and high angle boundaries. Mishin et al. ${ }^{8)}$ demonstrated the use of EBSD to analyze local heterogeneity in deformed metals. They suggested a simple and effective approach by consid- 
ering the microstructure as a two-phase mixture containing both high misorientation regions (HMRs) and low misorientation regions (LMRs), and successfully constructed the LMR/HMR map even when the specific characteristics of the low angle boundaries in the microstructure could not be completely identified. ${ }^{8)}$ The same approach has been adopted in the present study. The HMRs were considered as regions containing relatively higher stored energy in the specimen, where the recrystallization was observed to proceed preferentially.

One more concern in the EBSD analyses is that most analyses on recrystallization are carried out on post-mortem specimens, which could not provide direct relation between recrystallized grain and its precedent deformed structure. This problem might be solved by performing an in situ EBSD analysis in concurrent with the recrystallization process, which however is not practical at the present time because relatively long time is required to acquire the data over a large area. A recent study showed that the step size must be sacrificed in the in-situ EBSD analysis.9) Alternatively, to directly correlate the RX nuclei to the corresponding CR structure is feasible by carefully marking the specimen with micro-indentation. However, establishing a correlation between the $\mathrm{CR}$ and $\mathrm{RX}$ microstructures is difficult because the deformed substructures typically have sizes on the submicrometric scale. Step sizes of $0.03-0.1 \mu \mathrm{m}$ are required in order to accurately identify their misorientations relative to the surrounding matrix. An OIM measurement performed using the traditional approach to construct subboundaries is extremely time-consuming. ${ }^{10)}$ Therefore, a substitute approach for characterizing the misorientation of substructure is required.

In this study, the same area in both the CR and RX states of an electrical steel was examined without sacrificing the step size or integration time, and the RX nuclei were directly correlated to their precedent structures in the CR state. Moreover, we will demonstrate that the potential RX nucleation sites in the CR structure can be identified by applying a combination of KAM and HMR methods to the EBSD analyses. The texture of the deformed substructures which fulfil both high-KAM and HMR requirements correlates well to the texture of the $\mathrm{RX}$ grains.

\section{Experimental}

The chemical composition (wt $\%$ ) of the electrical steel used is: $0.0015 \mathrm{C}, 0.86 \mathrm{Si}, 0.31 \mathrm{Mn}, 0.22 \mathrm{Al}, 0.0021 \mathrm{~N}$, $0.005 \mathrm{P}$ and $0.005 \mathrm{~S}$. The steel was hot rolled from $100 \mathrm{~mm}$ to $12 \mathrm{~mm}$ thick with a finishing temperature of $1133 \mathrm{~K}$ and annealed at $1013 \mathrm{~K}$ for one hour. The average grain size of the hot band was measured to be $48 \mu \mathrm{m}$. The hot-rolled plate was machined to $10 \mathrm{~mm}$ thick followed by cold rolling to 3 mm thick, corresponding to a reduction of $70 \%$. Two pieces of CR sheet were annealed separately at $883 \mathrm{~K}$ and $903 \mathrm{~K}$ to obtain partial recrystallization, $\sim 5 \%$ and $\sim 20 \%$, respectively. The annealing was performed with a heating rate of $3 \mathrm{~K} / \mathrm{s}$ and $60 \mathrm{~s}$ holding time. Macrotexture was measured on the ND plane of the samples by X-ray diffraction (XRD) with $\mathrm{Co}-\mathrm{K} \alpha$ radiation. The orientation distribution functions (ODFs) were calculated from three pole figures, (110), (200) and (211), using the series expansion method.
Microtexture measurements were carried out in the transverse direction (TD) using EBSD technique. The EBSD analyses were conducted at $20 \mathrm{kV}$ using a Zeiss Supra 55 field-emission scanning electron microscope equipped with an Oxford Nordlys detector and Channel 5 software. The samples were electropolished to get a mirror-bright surface in a solution consisting of $20 \%$ perchloric acid and $80 \%$ glacial acetic acid at room temperature. In EBSD mapping, a step size of $0.25 \mu \mathrm{m}$ was used to scan four areas, each of them was of approximately $230 \times 172 \mu \mathrm{m}^{2}$. The indexed rates of the EBSD patterns were approximately 95\%. The local misorientation distribution in the $\mathrm{CR}$ structure was characterized using the KAM method ${ }^{7)}$ with a $3 \times 3$ mesh. The KAM value assigned to each pixel depends on the misorientation of the surrounding boundaries, and the step size. It was found that the optimal step size shall be approximately the average subgrain size, considering both spatial resolution and analysing time. The step size of $0.25 \mu \mathrm{m}$ was accordingly chosen based on the TEM observation of an IF steel. ${ }^{1)}$ Here, KAM $>10^{\circ}$ is taken as high-KAM.

The procedures used to obtain LMR/HMR maps are given in the following. Basically, the approach is to identify LMRs and the rest of the regions would be HMRs. Two parameters, $\theta_{\mathrm{o}}$ and $\mathrm{A}_{\mathrm{L}}$, are used to define a LMR. ${ }^{8}$ )

(1) $\theta_{0}$ defines the maximum misorientation between neighboring pixels within a LMR. In a LMR, the misorientations of all intragranular boundaries are lower than the selected value, $\theta_{0}$. The value of $\theta_{0}$ is used to separate low and high misorientation area. Therefore, $5^{\circ}-7^{\circ}$ should be a proper choice.

(2) $A_{L}$ is the minimum size of LMR, and it can be used to characterize the heterogeneity of the deformed microstructure on the meso-scale. The basic microstructural units in deformed structure are cells/subgrains. The size of cells/ subgrains are approximately uniform, but they are bounded by boundaries with varying characteristics, e.g. low misorientation angle versus high misorientation angle. The evolution of low angle boundaries to HABs is a continual process in plastic deformation, but the spatial distribution of these boundaries is non-uniform on the meso-scale.

The relative fraction of LMR and HMR depends on the selection of $\theta_{0}$ and $\mathrm{A}_{\mathrm{L}}$. The procedures for selecting $\mathrm{A}_{\mathrm{L}}$ are:

(1) The first step is to perform grain re-construction. In this work, $\theta_{0}$ is selected as $5^{\circ}$ to define the grains.

(2) The distribution of grain area as a function of grain size is thus obtained as shown in Fig. 1, revealing a bimodal distribution. The area fraction of the "normal grains" follows the Gaussian distribution with respect to the logarithm of the grain size.

(3) The LMRs, defined by $A_{L}$, are considered as "grains of abnormally large size" as compared to the normal grains. Therefore, $A_{L}$ is determined from Fig. 1 as $8 \mu \mathrm{m}$ in this work.

\section{Results and Discussion}

The rolling texture represented by the $\varphi 2=45^{\circ}$ ODF section derived from XRD pole figures is shown in Fig. 2. It is characterized by a strong $\alpha$-fiber, mainly from $\{001\}<110>$ through $\{112\}<110>$ to $\{111\}<110>$, and a moderate $\gamma$-fiber $(\{111\} / / N D)$. Figure 3(a) shows an OIM in ND of 


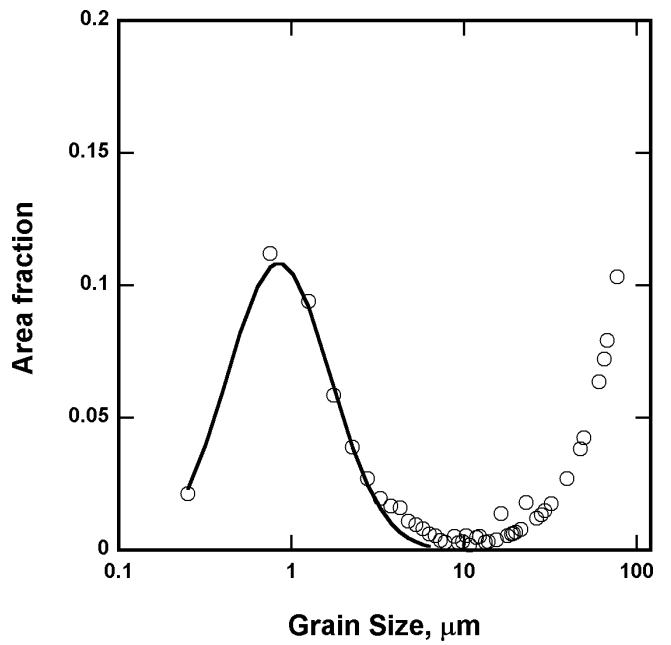

Fig. 1. The distribution of grain area as a function of grain size by re-construction of the deformed OIM using $5^{\circ}$ to define the "grains".

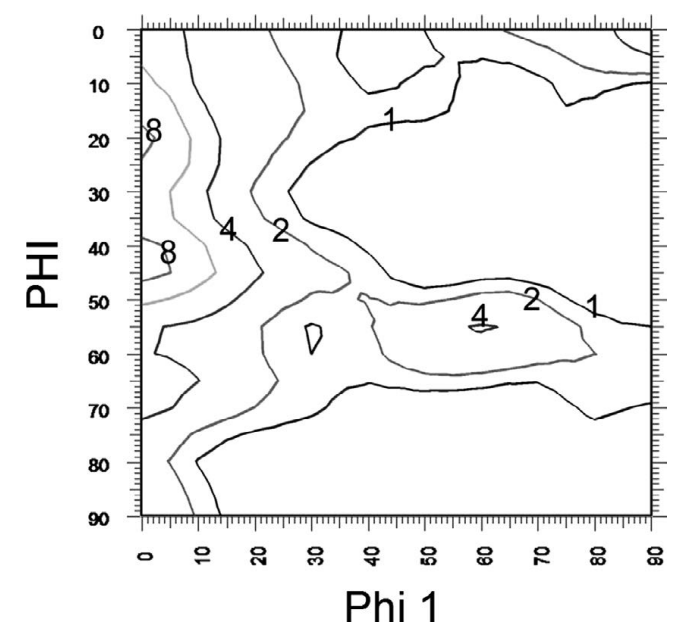

Fig. 2. ODF ( $\varphi 2=45^{\circ}$ section) derived from X-ray pole figures of the as cold-rolled sample. the as-rolled sample. The distribution of HABs in the same area of Fig. 3(a) is given in Fig. 3(b), which reveals the heterogeneity of deformed structure. Based upon the distribution and density of HABs, the deformed structure could be classified into three types.

Type A: The deformation substructures are rather homogeneous and nearly free of intragranular HABs.

Type B: Intragranular HABs are present with a moderate density and often aligned themselves in one direction or in two intersecting directions.

Type C: High density of HABs exists to subdivide the grain into domains of widely spread orientations.

The three types of structures are labelled in Fig. 3(b) accordingly. Examining the OIM closely, one can find that the crystal orientation varies gradually across a Type-A grain, whereas in Type-B structure, intragranular HABs are associated with micro-shear bands consisting of subgrains with similar orientations. With the aid of HMR analysis, areas containing high density of HABs in the deformed structure could be screened out as shown in Fig. 3(c). It was observed that Type-A grains are nearly free of HMRs, Type $\mathrm{C}$ grains are fully covered by HMRs, while Type B grains are partly covered by HMRs. Furthermore, the crystal orientations of HMRs were found to be mainly in the $\gamma$-fiber orientations with strong intensity near $\{111\}<112>$.

Figure 3(d) shows a color map highlighting the recrystallized grains of five texture components in the same area after annealing at $903 \mathrm{~K}$, with a deviation of $15^{\circ}$ being allowed for each component. The figure indicates that the Type-C grains are almost replaced by recrystallized grains with mostly cube and random orientations. The Type-B structure is partly covered by recrystallized grains, whereas the Type-A structure remains in the deformed state. These observations clearly show the correlation between recrystallization nucleation and the distribution of HABs in the deformed structure, and they are consistent with the finding on nucleation site distribution in a recent study on a bake-

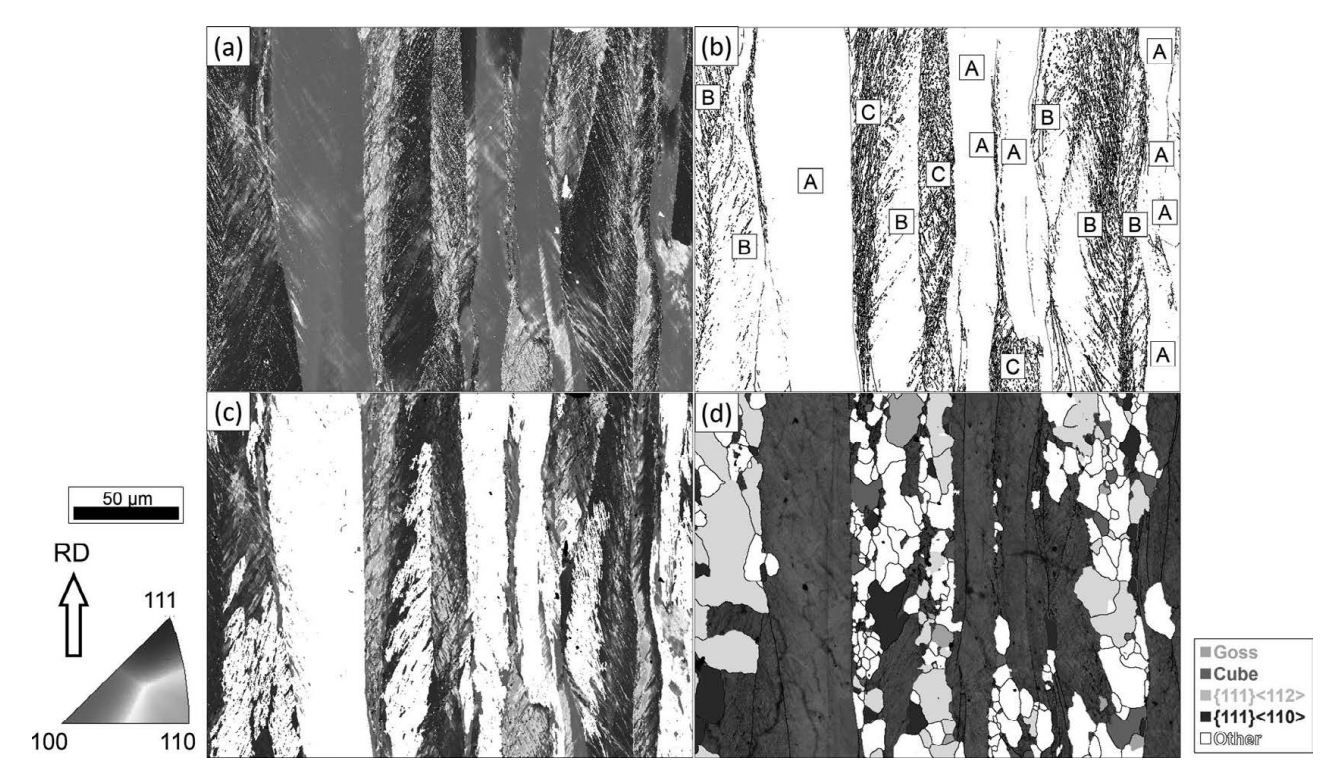

Fig. 3. (a) ND OIM of the as cold-rolled sample, (b) distribution of HABs $\left(>15^{\circ}\right)$ in (a), (c) HMR map of the same area in (a). (d) Color map, overlapped with EBSD image quality map, highlighting the recrystallized grains in the same area after annealing at $903 \mathrm{~K}$. (light blue: $\{111\}<112>$, blue: $\{111\}<110>$, red: $\{100\}<001>$, green: $\{110\}<001>$ with a deviation of $15^{\circ}$, and white: other orientations). 
hardenable steel. ${ }^{9)}$

Based on many experimental observations, ${ }^{5,6)}$ it is believed that RX nuclei are preferentially evolved from subgrains surrounded by HABs. The substructure partly or entirely bounded by HABs can be screened by the KAM method. ${ }^{7)}$ and the high-KAM areas in the deformed structure should represent potential nucleation sites of recrystallization. Here, high-KAM refers to KAM values exceeding $10^{\circ}$. To confirm this argument, a specimen annealed at $883 \mathrm{~K}$ to yield an RX fraction of approximately 5\% was used to determine whether a close connection exists between the recrystallized nuclei and the corresponding CR structure. Examples are presented in Figs. 4 and 5. In Fig. 4(a), parallel microbands that mainly show a Goss orientation are present in a $\gamma$-fiber matrix of a Type-B grain, and they are generally bounded by intragranular HABs (black lines). The KAM map in Fig. 4(b) confirms that most of the highKAM areas are adjacent to the HABs. Figure 4(c) shows the OIM of the partly recrystallized sample, clearly indicating that the recrystallized nuclei originate from the high-KAM substructures, as expected. A (200) pole figure of the CR structure with $\mathrm{KAM}>10^{\circ}$ in the area corresponding to the recrystallized grains is shown in Fig. 4(d), revealing that the orientations of the recrystallized grains coincide well with the orientation of the high-KAM areas.

Another example of the formation of recrystallized grains is given in Fig. 5. The OIM in Fig. 5(a) displays a CR microstructure/texture in which a Type-C region is situated between Type-A and Type-B regions. The central Type-C region contains a high density of HABs and, therefore, high-KAM areas. After the CR sample was annealed, eleven recrystallized grains were found in the Type-C region. Figure 5(b) shows the position and orientation of the eleven recrystallized grains. The distribution and orientation of the high-KAM areas in this region is given in Fig. 5(c). Only a few high-KAM areas are observed in the Type-B region. Ten of eleven recrystallized grains in Fig. 5(b) exhibit oneon-one orientation correspondences with the high-KAM

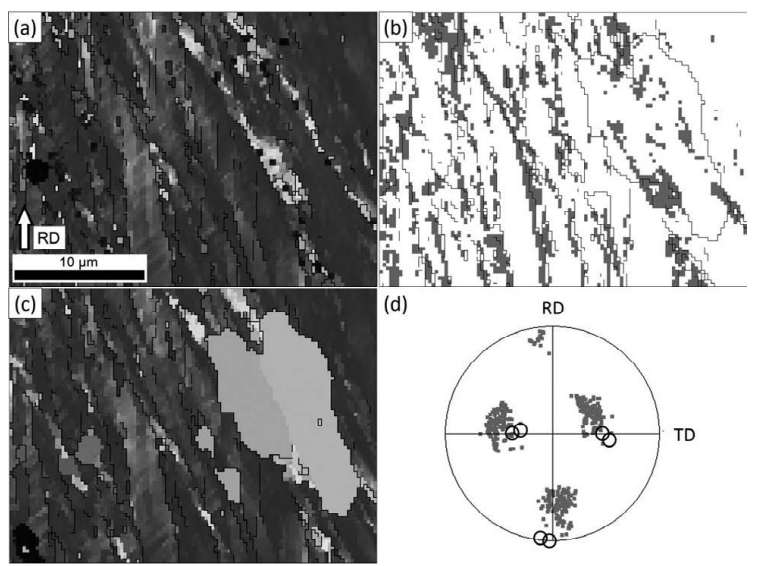

Fig. 4. Formation of recrystallized grains in Type-B structure. (a) ND OIM of the cold-rolled structure, in which HABs $\left(>15^{\circ}\right)$ are highlighted in dark lines, (b) map of high-KAM pixels, (c) ND OIM of the partly recrystallized state, and (d) (200) pole figure of the high-KAM pixels in (b) in the area corresponding to the recrystallized grains. The black circles correspond the $\{100\}$ poles of the recrystallized grains. The sample was annealed at $883 \mathrm{~K}$. areas in the CR structure. These examples provide direct evidence of the evolution of recrystallized grains at the highKAM sites in the CR structure.

The ODF section $\left(\varphi 2=45^{\circ}\right)$ of the high-KAM areas in the CR structure are shown in Fig. 6(a). In the ODF section, the intensity is mainly concentrated near $\{111\}<112>$ as well as $\{100\}<110>$ to $\{112\}<110>$, and weak intensities are observed near $\{110\}<100>$ and $\{100\}<001>$. The highKAM areas oriented from $\{110\}<100>$ to $\{100\}<001>$ are located at the grain boundaries of the Type-A grains as revealed in Fig. 5(c). These high-KAM areas, though surrounded by HABs, have low stored energies and do not develop into RX nuclei during annealing. They should be

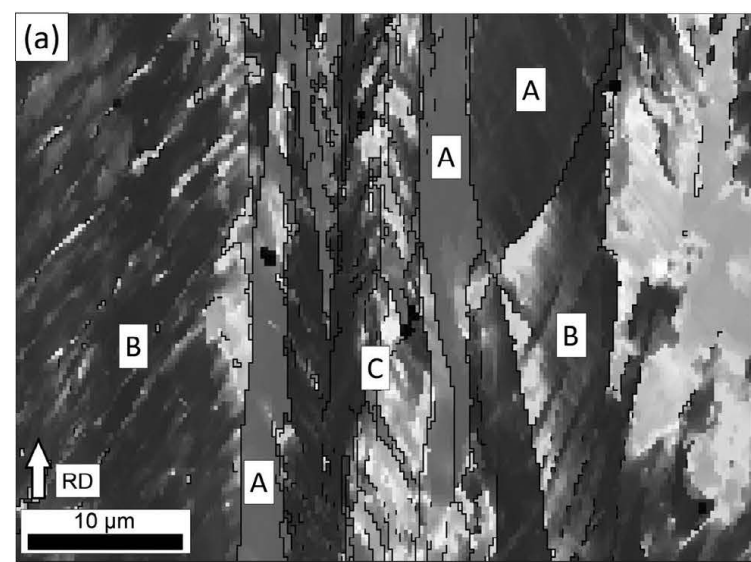

(b)
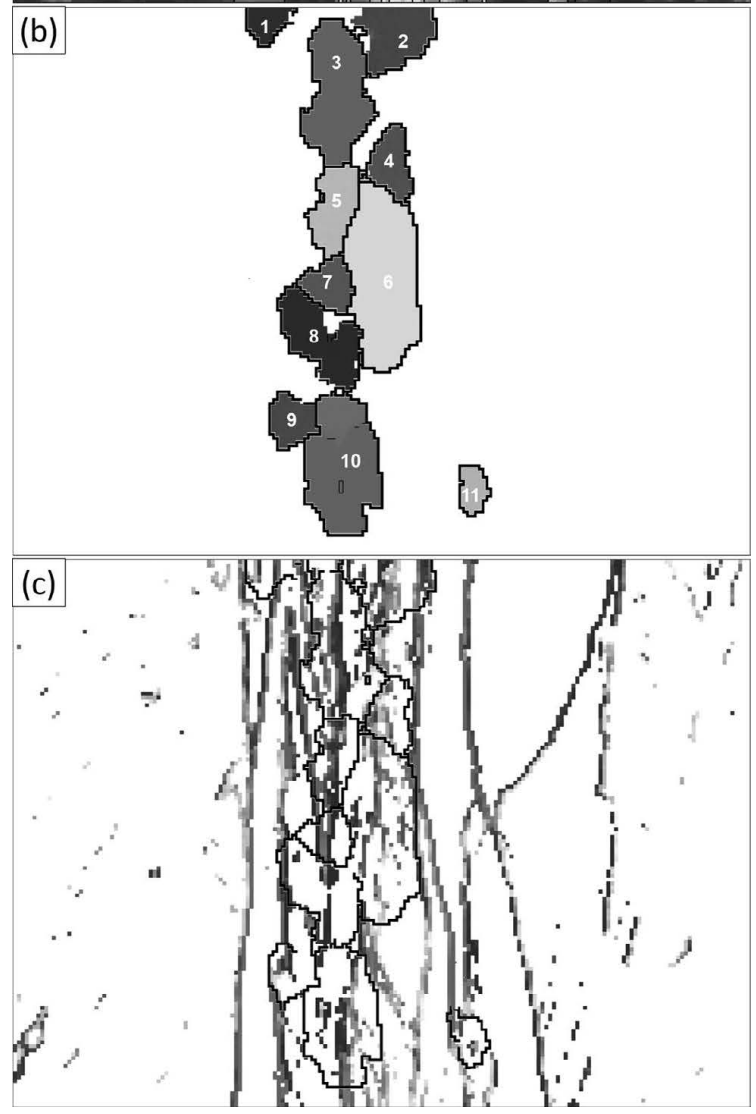

Fig. 5. Formation of recrystallized grains in Type-C structure. (a) ND OIM of the cold-rolled structure, in which HABs $\left(>15^{\circ}\right)$ are highlighted in dark lines, (b) OIM of the recrystallized grains, and (c) OIM of high-KAM pixels, in which the boundaries of the recrystallized grains are highlighted in dark lines. The sample was annealed at $883 \mathrm{~K}$. 
(a)

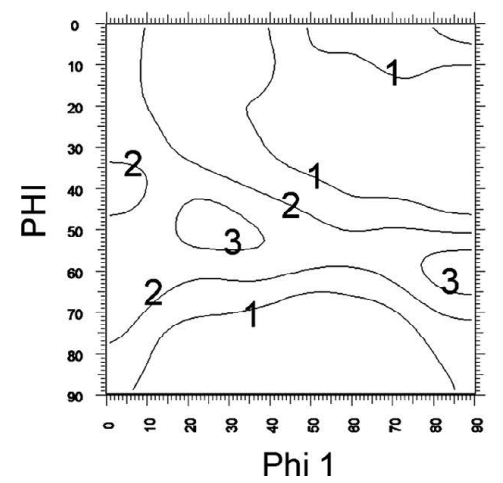

(b)

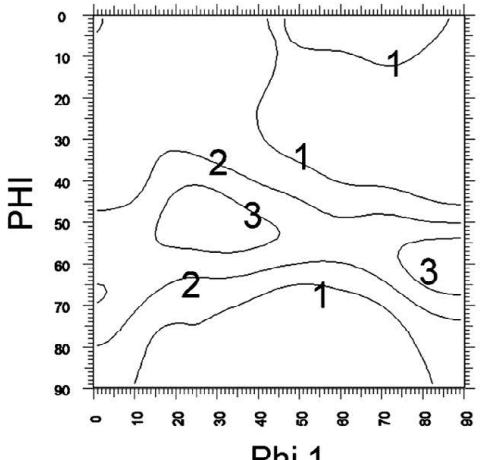

(c)

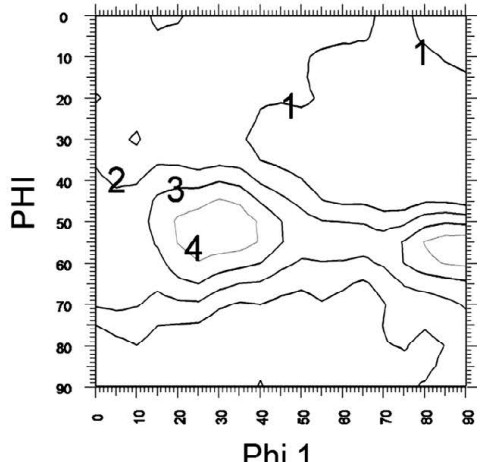

Fig. 6. ODF $\varphi 2=45^{\circ}$ section of (a) the high-KAM areas in cold-rolled sample (EBSD), (b) the areas fulfilling both highKAM and HMR (EBSD) and (c) the fully recrystallized sample (XRD) annealed at $983 \mathrm{~K}$.

excluded accordingly. This is accomplished by obtaining the ODF section $\left(\varphi 2=45^{\circ}\right)$ of the areas fulfilling both requirements of high-KAM and HMR as shown in Fig. 6(b), since the HMR analysis highlights areas having high stored energies. The intensities along partial $\alpha$-fiber in the high-KAM ODF (Fig. 6(a)) are much reduced in Fig. 6(b). In other words, a great portion of the high-KAM areas exhibiting orientations of the partial $\alpha$-fiber do not belong to HMRs, but are located at the original high angle grain boundaries. The ODF of the fully recrystallized sample calculated from XRD pole figures is shown in Fig. 6(c). The ODF of areas meeting both criteria of high-KAM and HMR in the CR structure (Fig. 6(b)) exhibits a high degree of similarity to the ODF of the fully recrystallized sample (Fig. 6(c)). It has to be emphasized that the RX texture is not necessary to be exactly the same as that of the RX nuclei (or areas fulfilling both criteria of high-KAM and HMR), since the competition of the RX grains in growth also play an important role in determining the RX texture. However, the present result confirms that the recrystallized grains must be evolved from the areas having high local misorientation in the CR structure, which fulfilling both criteria of high-KAM and HMR. This work clearly demonstrates that the combination of both KAM and HMR methods in the EBSD analysis not only sheds light on studying the evolution of the local microstructure and texture but also is capable of predicting the recrystallization texture to a certain extent based on the texture of local substructures in the as-deformed state.

\section{Summary}

In this study, the same area in both CR and partially $\mathrm{RX}$ states was analyzed using EBSD to obtain the correlation between RX nuclei and CR substructure. The local misorientation of substructure was characterized by using both the kernel average misorientation (KAM) and the high misorientation region (HMR) analyses. The RX nuclei in partially recrystallized specimen were found to form preferentially in the CR substructure having high local misorientation. A one-on-one correlation is therefore established between the RX nuclei and the high-KAM/HMR areas. Moreover, the orientation distribution of the areas having high local misorientation in the CR structure, which fulfil both criteria of high-KAM and HMR, was found to have a great similarity to that of recrystallized grains, indicating a possibility of predicting RX texture from the deformed substructure.

\section{Acknowledgement}

This work is supported by the Ministry of Science and Technology, R. O. C. (grant number NSC-1022216-E-006-032 and MOST-103-2622-E-006-037). The NSYSU team also acknowledges the support of the China Steel Corporation.

\section{REFERENCES}

1) B. L. Li, A. Godfrey, Q. C. Meng, Q. Liu and N. Hansen: Acta Mater., 52 (2004), 1069.

2) B. Hutchinson: Phil. Trans. R. Soc. London A, 357 (1999), 1471.

3) L. Kestens, J. J. Jonas, P. V. Houtte and E. Aernoudt: Metall. Mater. Trans. A, 27A (1996), 2347.

4) H. G. Kang, K. M. Lee, M. Y. Huh, J. S. Kim, J. T. Park and O. Engler: J. Magn. Magn. Mater., 23 (2011), 2248.

5) F. J. Humphreys and M. Hatherly: Recrystallization and Related Annealing Phenomena, 2nd ed., Elsevier, Amsterdam, (2004), 213.

6) R. D. Doherty, D. A. Hughes, F. J. Humphreys, J. J. Jonas, D. J. Jensen, M. E. Kassner, W. E. King, T. R. McNelley, H. J. McQueen and A. D. Rollett: Mater. Sci. Eng., A238 (1997), 219.

7) L. N. Brewer, D. P. Field and C. C. Merriman: Electron Backscatter Diffraction in Materials Science, 2nd ed., ed. by A. J. Schwartz, M. Kumar, B. L. Adams and D. P. Field, Springer, New York, (2000), 251.

8) O. V. Mishin, A. Godfrey and D. J. Jensen: Electron Backscatter Diffraction in Materials Science, 2nd ed., ed. by A. J. Schwartz, M. Kumar, B. L. Adams and D. P. Field, Springer, New York, (2000), 263.

9) D. I. Kim, J. S. Kim, J. H. Kim and S. H. Choi: Acta Mater., 68 (2014), 9.

10) V. Randle and O. Engler: Introduction to Texture Analysis: Macrotexture, Microtexture and Orientation Mapping, CRC Press, New York, (2000), 245. 